\title{
Manejo oportuno de paciente con hipoplasia maxilar y agenesia dental. Reporte de Caso Clínico
}

\author{
Mateo Rondón Santa ${ }^{1}$, Nelson Eduar León², Juan Pablo Monsalve Guerrero ${ }^{3}$
}

RESUMEN Introducción: el propósito de este estudio es hacer una revisión de literatura y presentar un caso clínico para el majeo oportuno de pacientes con hipoplasia del maxilar y agenesia dental. Se describe el caso clínico de un paciente pediátrico de 8 años de edad que presenta hipoplasia maxilar y agenesia dental múltiple, el cual acude a clínica de mediana complejidad pediátrica de la Fundación Universitaria del Área Andina Seccional Pereira, Colombia, por presentar retraso en la exfoliación de su dentición temporal. Para elegir el tratamiento adecuado para este caso, se realiza revisión bibliográfica y se opta por el uso de tornillo Hyrax acompañado de máscara facial; se lograron notables resultados visibles que permitieron mejorar la estética y función en el paciente.

Método: revisión de literatura; tornillo Hyrax y máscara facial.

Resultados: el resultado de este tratamiento oportuno que se le brindó al paciente, fue conseguir una descruzada de las arcadas superior e inferior significativamente, debido al tratamiento ortopédico: la fuerza ejercida por el tornillo Hyrax y la máscara facial, lograron una mordida borde a borde, la cual permitió mejorar la masticación del paciente y a su vez la apariencia física cambió notablemente y un poco más asimétrico que al principio.

Conclusiones: este es un procedimiento terapéutico eficiente, ya que con él se logra un rápido ensanchamiento de las bases óseas del maxilar, permitiendo aumentar la longitud del arco dentario y el espacio necesario para la alineación y futura implantación de los dientes ausentes, teniendo a favor la temprana intervención que permitió enfrentar suturas maleables.

Agenesia Dental;

PALABRAS Hipoplasia Maxilar;

CLAVE Máscara Facial;

Oligodoncia.

1 Odontología, noveno semestre, Fundación Universitaria del Área Andina, mrondon4@estudiantes.areandina.edu.co,nleon11@estudiantes.areandina.edu.co, iguerrero25@estudiantes.areandina.edu.co. 


\title{
Timely management of patients with maxillary hypoplasia and dental agenesia. Clinical Case Report
}

\begin{abstract}
Introduction: the purpose of this study was to make a review of the literature and present a clinical case for the opportunity opportunity of patients with maxillary hypoplasia and dental agenesis, describe the clinical case of an 8 -year-old pediatric patient, who presents maxillary hypoplasia and multiple dental agenesis which goes to a clinic of medium pediatric complexity of the University Foundation of the Andean Area headquarters Pereira, Colombia, for presenting delayed exfoliation of its temporary dentition, the literature review is performed to choose the appropriate treatment.
\end{abstract}

Method: this case We opted for the use of Hyrax screw accompanied by a facial mask, remarkable visible results were achieved that allowed to improve the aesthetics and function in the patient. Methods: Clinical case report - Literature review

Results: the result of this timely treatment that was given to the patient was to achieve a description of the upper and lower arches potentially, this due to orthopedic treatment and the forces exerted by the Hyrax screw and the facial mask, achieving an edge-to-edge bite, the quality improve the chewing of the patient, in turn the physical appearance took a noticeable change and a little more asymmetric than at the beginning.

Conclusions: This is an efficient therapeutic procedure, since with this a rapid widening of the bases abroad is achieved, increasing the length of the dental arch and the space necessary for the alignment and future implantation of the missing teeth, having a favor Early intervention that occurred faced with malleable sutures.

Dental Agenesis;

Maxillary Hypoplasia;

KEYWORDS Hyrax Screw;

Facial Mask,

Oligodontics. 


\section{INTRODUCCIÓN}

La hipoplasia maxilar se caracteriza por una deficiencia en el desarrollo sagital, vertical y transversal del hueso maxilar, esto conlleva a maloclusiones clase 111 que se presentan desde temprana edad, con distintas causas y de diferentes maneras (1).

La expansión rápida ortopédica maxilar se ha practicado desde hace varios años, cuando fueron presentados los primeros disyuntores y sin importar que estos eran más aparatosos, son similares a los que se utilizan actualmente para el tratamiento de este tipo de maloclusiones. (2)

Por otro lado, la agenesia dental es una patología congénita que consiste en la ausencia de una o más piezas dentarias, por lo general de origen genético o que pueden ocurrir por condiciones locales o ser manifestación de alteraciones sistémicas. Se producen como consecuencia de transformaciones que interfieren en el proceso normal de la odontogénesis $\mathrm{y}$, dependiendo de la etapa en la que ocurran, pueden verse comprometidas la dentición primaria, la permanente o ambas. (3)

Según Lewis y Davis, las anomalías dentarias se clasifican en: I. de número, II. de forma, III. de estructura y textura, IV. de color, V. de erupción y exfoliación, y VI. de posición. En este caso vemos que se trata de anomalías de número, las cuales se originan en la fase de formación de la lámina dental. Hace referencia al incremento o falta de piezas dentarias en cualquiera de los dos arcos dentarios. En nuestro caso en particular hablamos de oligodoncia, referida a la ausencia clínica y radiológica de más de seis dientes. (4)(5)

\section{METODOLOGÍA}

Reporte de caso clínico - Revisión de literatura.

Caso clínico

Paciente pediátrico de 9 años de edad, definitivamente positivo, originario de la ciudad de Pereira, Risaralda, quien acude a consulta odontológica a clínica universitaria de mediana complejidad; su motivo de consulta era por revisión de rutina. Se realiza la apertura de historia clínica al examen clínico extraoral, y se encuentra que presenta asimetría en sus tercios faciales, porque su tercio medio está disminuido y su perfil cóncavo, debido a su prognatismo mandibular.

Al examen clínico intraoral mostraba normalidad en sus tejidos blandos, y al examen dental se encontró con una mordida cruzada anterior y ausencia de dientes 55, 51, 61, 65,75 y 85; su acudiente manifiesta que dichos dientes nunca erupcionaron.

Para confirmar diagnóstico de agenesia se solicita radiografía panorámica, encontrándose ausencia de gérmenes dentarios de múltiples unidades dentarias, 18, 
$17,16,13,23,26,27,28,38,37,36,35,32,42,45,46,47$ y 48 . También presenta disminución notable en el tamaño del maxilar superior, que hace al paciente con asimetría facial y su perfil convexo.

Para el tratamiento se inició con su fase higiénica; luego se investiga en la literatura el tratamiento más adecuado y se opta por tornillo Hyrax acompañado con máscara facial para lograr una expansión del maxilar, aprovechando su edad, donde las suturas circunmaxilares aún son maleables. Para su instalación se siguieron los siguientes requisitos expuestos en las bibliografías:

- Colocarlo lo más profundo posible en el centro del paladar, separado de la mucosa palatina a $3 \mathrm{~mm}$ aproximadamente.

- Guiar los alambres conectores hacia las bandas, luego doblarlos y unirlos entre los caninos y primeros molares temporales, con el objeto de lograr una máxima estabilidad del aparato.

- Evitar el contacto del tornillo y los alambres conectores con el tejido gingival.

\section{RESULTADOS ESPERADOS}

- Determinar un tratamiento eficaz para paciente pediátrico con hipoplasia maxilar.

- Descruzar la mordida en paciente clase 111.

- Identificar rápidamente el número de dientes perdidos debido a la agenesia dental mixta, y así llevar a cabo un tratamiento oportuno, una vez erupcionen todos los dientes permanentes.

Con el tratamiento y la función que se busca lograr con el tornillo Hyrax y la máscara facial, está expandir el maxilar superior y el paladar en sentido lateral y anteroposterior, antes de que las sutura circunmaxilar y la sutura media palatina, alcance su etapa de osificación, lo cual sucede a los 12 años. Este proceso de expansión rápida palatina tomará 16 meses para que el paciente quede con la mordida descruzada y así recupere su función masticatoria, fonética y deglución fisiológica con normalidad.

Una vez esto suceda, la autoestima y la personalidad de estos pacientes cambia significativamente, ya que la apariencia física mejora en todos los sentidos.

\section{RESULTADOS OBTENIDOS}

El resultado de este tratamiento oportuno que se le brindó al paciente fue lograr una descruzada de las arcadas superior e inferior significativamente, debido al tratamiento 
ortopédico y a la fuerza ejercida por el tornillo Hyrax y la máscara facial, logrando una mordida borde a borde, la cual permitió mejorar la masticación del paciente, a su vez la apariencia física tomó un cambio notable y un poco más asimétrico que al principio.

\section{DISCUSIÓN}

La característica física más marcada en pacientes con hipoplasia maxilar y agenesia dental, es la asimetría facial en ellos, por lo cual en su infancia y adolescencia sufren bullying por parte de otros, y en su etapa de adulto su autoestima es baja en la gran mayoría de los casos debido a su anomalía congénita; se pueden creer inferiores al resto o con una apariencia física inadecuada, ya que en la sociedad actual en la mayoría de personas prima el valor de la belleza física, y por esto es sumamente importante el diagnóstico precoz, para así proceder al tratamiento más oportuno, adecuado, y sobre todo a una edad en la cual este sería más positivo y económico.

Una de las razones económicas más importantes para pacientes con estas anomalías congénitas, es saber que cuanto más temprano se diagnostiquen, es más económico y menos invasivo el tratamiento, debido a que, si esto sucede a temprana edad, el tratamiento oportuno será con aparatología ortopédica, la cual es más favorable económicamente, ya que los materiales no son tan costosos como un tratamiento quirúrgico invasivo y doloroso para un paciente adulto.

\section{CONCLUSIONES}

Todo odontólogo debe de estar en la capacidad de saber la importancia de diagnosticar rápidamente a un paciente con hipoplasia maxilar a temprana edad, antes de que se osifiquen las suturas circunmaxilar y la sutura media palatina, y hacer que el tratamiento ideal no sea invasivo, quirúrgico y tampoco tan costoso para el paciente.

Es indispensable para el paciente saber cómo se puede llegar a expandir el maxilar, y por medio de cuál aparatología se logra.

Así mismo, el odontólogo debe tener presente las anomalías dentarias o características que presenta un paciente con agenesia dental, debido a que es indispensable para el clínico conocerlas, ya que pueden llegar a estar asociadas a un síndrome o también a factores hereditarios, y no confundir dicha anomalía con otras irregularidades dentarias, para remitirlo con un rehabilitador oral y prestarle el tratamiento más eficaz para el paciente. 
1. Machado R, Bastidas M, Arias E, Quirós O. Disyunción maxilar con la utilización del expansor tipo Hyrax en pacientes con labio y paladar hendidos. Revisión de la Literatura. Rev Latin Ortod Odontol. 2012

2. Hathiram B.T., Khattar V.S. Unilateral maxillary sinus aplasia with ipsilateral rhinolith: A rare anomaly with a rare association Otorhinolaryngology Clinics, Volume 3, 2011

3. Quirós O. Haciendo Fácil la Ortodoncia. Cáliz: Editorial Amolca; 2012.p.42830.

4. Bog JR, Catalá M, García C, Mendoza A. Odontopediatría, Barcelona, España: Editorial Masson; 2005.

5. Meneses D, Botero P. Alternativas para el tratamiento de hipoplasias maxilares. CES Odonto [Internet]. 2012 [Consultado 25 Ene 2012]; 64/81. Disponible en: http://revistas.ces.edu.co/index.php/odontologia/article/view/2227/1477

6. Dra. Manuela Ricardo Reyes, Dra. Raquel Bibiana Comas Mirabent, Dra. Maira Raquel Martinez Ramos, Dra. Paula Mok Barcelo. Expansion rapida del maxilar con el tormillo Hyrax en un adolescente. Clinica estomatologica provincial [Internet]. 2014 [Consultado 19 Mar 2014]; 417. Disponible en: http://scielo. sld.cu/scielo.php?script=sci_arttext\&pid=S1029-30192015000300014

7. B.P. Fournier, M.H. Bruneau, S. Toupenay, S. Kerner, A. Berdal, V. CormierDaire, S. Hadj- Rabia, A.E. Coudert, M. de La Dure-Molla. Patterns of Dental Agenesis Highlight the Nature of the Causative Mutated Genes. Journal of Dental Research [Internet]. 2018; Vol. 97(12) 1306-1316.

8. Bashar Reyad Elmomani, Jumana Tbeshat, Ahmad Madallah Tarawneh, Zaid Al Zoubi, Abdullah Nazah. Alternate rapid maxillary expansions and constrictions technique used in treatment of class 111 malocclusion with macillary hypoplasia in an adolescent - case report. Pakistan Oral \& Dental Journal [Internet]. 2016 [Consultado Abr - Jun 2016]; Vol. 36(2).

9. Su Ji Choi, Je Woo Lee \& Ji Hyun Song. Dental anomaly patterns associated with tooth agenesis. [Internet]. 2017[Consultado 25 Ene 2017]; 161-165.

10. Lannefranque A, González S, Jofré S. Hipoplasia maxilar: experiencia clínica en criterios terapéuticos determinados por la maduración esqueletal / Maxillary hypoplasia: clinical experience in therapeutic criteria determined by the esqueletal maturation. Biblioteca virtual em saúde. [Internet].; 2004 [cited 2019 Abril 03. Disponible en: http://bases.bireme.br/cgi-bin/wxislind.exe/iah/ online/?IsisScript $=$ iah/iah. $x$ is $\&$ src $=$ google $\&$ base $=$ LILACS\&lang $=$ p\&nextAct ion=lnk\&exprSearch=476855\&indexSearch=ID.

11. Santander AM. Tratamiento de maloclusión clase III por hipoplasia maxilar, con mordida cruzada posterior; mordida abierta anterior y crecimiento vertical / Treatment of a patient with Class III maxillary hipoplasia, posterior crossbite(.). Biblioteca virtual en salud. [Internet].; 2003 [cited 2019 Abril 03. Disponible en: http://revistas.ces.edu.co/index.php/odontologia/article/view/544/315.

12. Maldonado Saucedo F, Gómez Pedroso Balandrano A, Sánchez Pozos V, Navarro Barrón AN. Osteotomía Le Fort I oblicua para el tratamiento de la hipoplasia. Redalyc. [Internet].; 2013 [cited 2019 Abril 03. Disponible en: https://www.redalyc.org/articulo.oa?id=47327854014.

13. Contemporary Clinical Dentistry. Assessment of the dental and skeletal effects of fan-type rapid maxillary expansion screw and Hyrax screw on craniofacial 


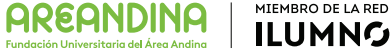

REFERENCIAS

structures. Contemporary Clinical Dentistry. [Internet].; 2017 [cited 2019 Abril 03. Disponible en: http://www.contempclindent.org/article.asp?issn=0976$237 \mathrm{X}$;year $=2017$; volume $=8 ;$ issue $=1 ;$ spage $=64$; epage $=70 ;$ aulast $=$ Gopalakrish nan

14. Díaz R, R. Agenesia en dentición permanente. SciELO. [Internet].; 2009 [cited 2019 Abril 03. Disponible en:http://www.scielo.org.co/scielo.php?script=sci arttext\&pid=S0124-00642009000600012\&lang=es.

15. Dean J. Trastornos adquiridos y del desarrollo de los dientes y las estructuras orales asociadas. In James E. Jones DMEP, editor. McDonald y Avery. Odontología pediátrica y del adolescente. Barcelona: Elsevier; 2016. p. 61. 


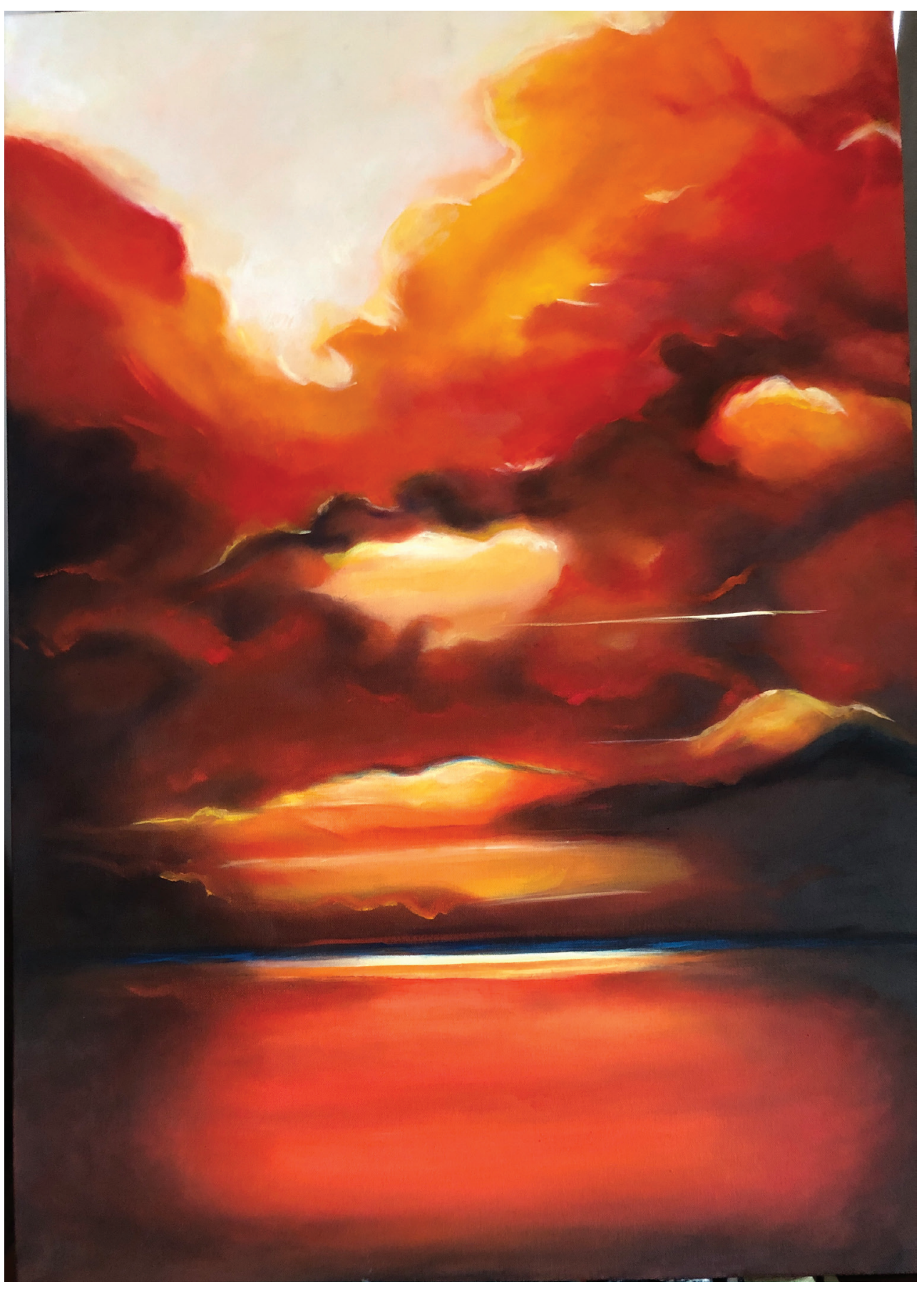

Oleo. “Atardecer pereirano”, Virginia Aristizábal Parra. 